\title{
Research Square \\ Constraints on the spatial and temporal variations of the gravitational constant from quasar spectra
}

T. D. Le ( $\sim$ leducthong@tdtu.edu.vn )

Ton Duc Thang University

\section{Research Article}

Keywords: quasars, varying-gravitational constant, absorption spectra analysis, GUTs

Posted Date: May 25th, 2021

DOI: https://doi.org/10.21203/rs.3.rs-546726/v1

License: (a) (i) This work is licensed under a Creative Commons Attribution 4.0 International License. Read Full License 


\title{
Constraints on the spatial and temporal variations of the gravitational constant from quasar spectra
}

\author{
T. D. Le $e^{1,2}$ \\ ${ }^{1}$ Division of Computational Physics, Institute for Computational Science, Ton Duc Thang \\ University, Ho Chi Minh City, Vietnam \\ ${ }^{2}$ Faculty of Applied Sciences, Ton Duc Thang University, Ho Chi Minh City, Vietnam \\ Email: leducthong@tdtu.edu.vn
}

\begin{abstract}
The possible variations of fundamental physical constants present a critical examination of grand unification theories (GUTs). The most useful way to investigate these variations could be based on observations that directly test them with a deep-look back in time in the universe. Using combinations of high-resolution spectra of quasars and Ritz wavelengths in the laboratory, we found an upper limit on a possible cosmological deviation of the gravitational $\dot{G} / G=(0.918 \pm 2.830) \times 10^{-15} \mathrm{yr}^{-1}$ over cosmic timescales.. This result may be considered as a new tool for checking the physical phenomena of the GUTs.
\end{abstract}

Keywords: quasars; varying-gravitational constant; absorption spectra analysis; GUTs

\section{Introduction}

One of the most significant tests of current physics is the parameters of unification scenarios. The ongoing efforts to achieve such hypotheses have reestablished the question of space or time variation of the fundamental physical constants. Answering this question, is important considering that the constancy of these constants, especially the gravitational constant $G$, has been suspected for a long time [1-5]. This sits along our immediate aims to unify the four forces in nature [6,7]. However, contemporary physics theories, including M-theory, string theory, and superstring theory [8], do not necessitate changing these constants. They have a natural way and self-consistent framework for their variations [9-10]. As a consequence, modern physics theories indicate that in the gauge couplings with a common three-dimensional subspace, the fine-structure constant $\alpha$, the proton-to-electron mass ratio $\mu$, and the gravitational constant $G$ would differ to the opposite square of the mean scale of the extra-dimensions. The evolution of these dimensions' scale size is linked with their space or time variations [11-14]. Furthermore, this has been recently confirmed by the change of $\alpha$ over cosmological space-time. The value of $\alpha$ was different at different times and epochs during the evolution of the universe $[15,16]$. Nevertheless, the variation of both $\alpha$ and $G$ with space or time is model-dependent, with a relationship as follows: $\dot{\alpha} / \alpha^{2} \sim \dot{G} / G$. Various studies have been conducted on the different values in $\alpha$, with different deep look-back times into the universe. Lately, most of the investigations have applied the issued Cosmic Microwave Background (CMB) anisotropy data to established spatial and temporal variations in $\alpha$ early of the universe [17-19].

Various studies have utilized high-resolution quasar spectra of absorption systems and found evidence for a smaller change of $\alpha$ with spanning redshifts of $0.5<\mathrm{z}<3.5$. If $\alpha, \mu$, and $G$ changed 
with the cosmic space-time, we could obtain their different values with different redshifts [20]. The measured effect of space or time variation of the gravitational constant should present a significant role in employing local measurements, for example, the sun, the solar system, or the solar neighborhood, and the from Big-Bang nucleosynthesis. Using Lunar Laser Ranging provided a reasonable stringent limit on the time-variation of the gravitational constant $\dot{G} / G=(0.2 \pm$ $0.7) \times 10^{-12} \mathrm{yr}^{-1}$ [21]. However, a better result was found $-0.3 \times 10^{-12} \mathrm{yr}^{-1} \leq \dot{G} / G \leq 0.4 \times$ $10^{-12} \mathrm{yr}^{-1}$ from the Big-Bang nucleosynthesis [22, 23]. Simultaneously, a weaker result used the Hubble diagram of Type Ia supernovae, giving $\dot{G} / G \sim 1 \times 10^{-11} \mathrm{yr}^{-1}$ at $z \sim 0.5$ [24, 25]. Updated results have improved this limit and reported $-(1.10 \pm 1.07) \times 10^{-12} \mathrm{yr}^{-1}<\dot{G} / G<0$ and the best result to date found $-0.6 \times 10^{-12} \mathrm{yr}^{-1} \leq \dot{G} / G<0$ [26-32]. As a result of these findings, a new technique used the data from astrophysical observations to detect the space-time variations of the fundamental constants, including the fine-structure constant and the proton-toelectron mass ratio. This technique has a significant advantage in that it could provide the most stringent constraints $\Delta \alpha / \alpha=(0.027 \pm 0.832) \times 10^{-6}$ for the fine-structure constant and $\Delta \mu / \mu=$ $(0.025 \pm 0.262) \times 10^{-7}$ for the proton-to-electron mass ratio with the evaluation of analytical and systematic errors with high precision [33-35].

The aims of this study use a combined wavelength of Ritz in the laboratory and [Fe II] lines from the quasar to search for the effects of space-time variation of the gravitational constant $G$. Our analysis yields an estimate for the possible cosmological deviation of the gravitational constant $\dot{G} / G=(0.918 \pm 2.830) \times 10^{-15} \mathrm{yr}^{-1}$. In any case, this result could significantly improve upon the previously published results [24-32, 36,37].

\section{2. $\dot{G} / G$ with Quasar Spectra}

The redshifted spectra of quasars provide an essential tool used to search for the possibilities of spatial and temporal variations of the fundamental dimensionless constants, such as the finestructure constant $\alpha$ and the proton-to-electron mass ratio, $\mu$ over cosmic timescales. These constants' sensitive changes could be identified from the measured spectra of observational systems with wide-ranges of redshifts, because the resonance states of ions and other molecules to their ground-state of transitions are in these systems. Accordingly, changes could be found by a comparison between observed values and their laboratory values. As a result, the separated energy levels associated with fine-splitting in terms of $\alpha^{4}$ and the maximum energy level were proportional to $\alpha^{2}$. Mainly, a kind of coupling with the scalar fields and various other fields is based on the general relativistic effects of the redshift $(z)$. It can be determined by the loss energy $(E)$ of a photon, such: $z=-\Delta E / E$. This fractional change energy would be known as a fractional change in wavelength observations $-\Delta E / E=\Delta \lambda / \lambda \sim \Delta \alpha / \alpha$ [38]. Consequently, the effect of changes in $\alpha$ can directly be detected in any space or timescale of the universe. In this case, one can infer by a comparison of the observed wavelengths with their laboratory values to find the $\alpha$ variation [33-37]. Based on GUTs, spatial or temporal variation in the fundamental physical constants can realize the aim to unify gravitational and electromagnetic forces. Transmutable dimensions use to determine the weak scale, and all the related Yukawa couplings variation is the same. Therefore, we can hypothesize that the varied couplings are driven by a dilaton-type. This assumes that these variations will lead to the fine-structure constant and the Quantum 
Chromodynamics (QCD) scale, $(\triangle \Lambda Q C D) / \Lambda Q C D=R \Delta \alpha / \alpha$, here, $\Lambda_{Q C D}$ is QCD scale and $R$ can be determined by GUT. On the other hand, we can identify the $R$ value by model-independence at the low energy due to the relation $\alpha\left(M_{G U T}\right)=\alpha_{S}\left(M_{G U T}\right)$. Moreover, based on the dimensional transmutation in the weak scale, we can identify that the largest changes in Yukawa coupling $(h)$ will cause the Higgs vacuum expectation value $(v)$ changes. In this case, the $v$ value can be determined: $v=M_{\text {Planck }} \exp \left(-\left(8 \pi^{2 c}\right) / h^{2}\right)$ and $\Delta v / v=16 \pi^{2} c(\Delta h / h)=S(\Delta h / h)$ at the Planck scale based on the GUTs of mass. Then we can lead to $\Delta v / v=S(\Delta h / h)$, where $S \equiv$ $d \ln v / d \ln h, c \simeq h \simeq 1$, and $\Delta h / h=(1 / 2) \Delta \alpha / \alpha$. We can also find the electron mass and the proton-to-electron mass ratio variations as follows: $\Delta m_{e} / m_{e}=1 / 2(1+S) \Delta \alpha / \alpha$ and $\Delta m_{p} / m_{p}=$ $[0.8 R+0.2(1+S)(\Delta \alpha / \alpha)]$. Based on the perturbative approach, we can obtain the neutron mass $m_{n}$ and the average nucleon mass $m_{N}$ variations: approach $\Delta m_{n} / m_{n}=\Delta m_{N} / m_{N}=\Delta m_{p} / m_{p}$ [33-39]. Finally, we can easily infer different changes for both $\alpha$ and $G$ over cosmological spacetime. The probability of these changes could be examined by the relationship between them as follows [40].

$$
\frac{\Delta G}{G}=[1.6 R+0.4(1+S)] \frac{\Delta \alpha}{\alpha}
$$

Here, the free phenomenological parameters $(R, S)$ are related to QCD and EW sectors and, known as dimensionless couplings. Their values have differences based on different models. In the different models, laboratory tests are determined with the same or opposite signs, and then they are used in astrophysical observations. Based on the determined values such as $R=273 \pm 86$ and $S=630 \pm 230$, one can be applied to test the effect of space or time-variation in both $\alpha$ and $\mu$ in a higher precision way. This study examines the combined Ritz wavelengths and observed wavelengths from quasar spectra using previous data [41-46]. Wavelength calibration and analytical and systematic errors were detailed in these studies [41-46]. The [Fe II] transition wavelengths were used as a good candidate for this study, because they are often found in quasar spectra and are highly sensitive to variation in some physical dimensionless constants, for example the fine-structure constant, the proton-to-electron mass ratio, and the gravitational constant more than [C I] and [O I] lines [47]. The benefit of [Fe II] lines is that the definition of all parameters used for analysis has the same shape of line. Moreover, they eliminate all systematic effects because their ionization structure or observed substructure is medium compared with other ions that have been used, such as [C I] and [O I] lines [47]. The present analysis used a well-updated uncertainty error and high quality [Fe II] spectra [48-49]. Based on this uncertainty, the analysis process could estimate the systematic error with high accuracy. In the analysis practice, we used the combined wavelengths of 27 [Fe II] lines from quasar spectra and Ritz wavelengths from the laboratory. The energy levels of [Fe II] are determined from Fourier transform spectroscopy.

Our analysis identified that Doppler shift is used to determine the velocity scale of [Fe II] lines spectra from quasar observations. The selection of [Fe II] lines have the same velocities, the same shape, and they are narrow. We used Gaussian-fitting to determine the central of [Fe II] velocities and linewidths. The program was applied to single-fitting Gaussian for single-velocity components and multiple-fitting Gaussians for multiple-velocity components. Therefore, the characterizted components are based on the Doppler, column density- $N$, the absorption redshift- 
$z_{a b s}$, and the linewidth- $b$. Step by step, we also used the [Fe II] lines to estimate the $\Delta \alpha / \alpha$ values. Then, we used the $\Delta \alpha / \alpha$ values and combined with the fitting-parameters $(R, S, \Delta \alpha / \alpha)$ to determine the values of $\dot{G} / G$. Our analysis used the non-linear least-squares including a laboratory with an uncertainty of $10^{-6}$, and observed wavelength with an uncertainty of $10^{-7}$. In this way, we were able to estimate the effect of time-variation on the gravitational constant over cosmological timescales with high precision. In this way, the change values of $\dot{G} / G$ was applied only $\left(\chi^{2}\right)$ and minimum $\left(\chi_{\min }^{2}\right)$ with fitting-approximated reduction $\left(\chi^{2} \simeq 1\right)$. We also used $\Delta \chi^{2}=$ $\chi^{2}-\chi_{\min }^{2}=1$ to evaluate the variation of $\dot{G} / G$. We could then determine the maximal $\dot{G} / G$ changes due to $\Delta \chi^{2}=1$ for estimation of $\dot{G} / G$ errors. As a result, the expected result was derived by using minimal $\chi^{2}$ fitting each [Fe II] line, which was obtained an upper limit on the time variation of the gravitational constant $\dot{G} / G=(0.918 \pm 2.830) \times 10^{-15} \mathrm{yr}^{-1}$ (Table 1) and $\sigma_{\text {tot }}^{2}=\sigma_{\dot{G} / G}^{2}+\sigma_{\text {sys }}^{2}$ used to estimate the statistical and systematic errors. The illustration of the $G$ variation with redshift is shown in Figure 1.

Table 1: $\dot{G} / G$ was determined with redshift from the [Fe II] lines. The obtained result is $\dot{G} / G=(0.918 \pm 2.830) \times 10^{-15} \mathrm{yr}^{-1}$ with the weighted average of all lines. The lower level $3 d^{6}\left({ }^{5} \mathrm{aD}\right) 4 s^{6} \mathrm{D}_{9 / 2}$ was used for fitting all lines from quasars [33, 35, 41-45]. 


\section{PHL957 quasars}

\begin{tabular}{|c|c|c|c|c|c|}
\hline $3 d^{6}\left({ }^{5} z D\right) 4 p^{6} D_{9 / 2}$ & 2600.172 & 0.05000 & -0.01940 & -0.00891 & -1.90899 \\
\hline $3 d^{6}\left({ }^{5} z D\right) 4 p^{6} D_{7 / 2}$ & 2586.649 & 0.04197 & 0.05036 & 0.01285 & 1.28536 \\
\hline $3 d^{6}\left({ }^{5} z D\right) 4 p^{6} F_{11 / 2}$ & 2382.764 & 0.05876 & -0.01898 & -0.00872 & -1.71617 \\
\hline $3 \mathrm{~d}^{6}\left({ }^{5} \mathrm{zD}\right) 4 \mathrm{p}^{6} \mathrm{~F}_{9 / 2}$ & 2374.460 & 0.02106 & -0.27398 & -0.12581 & -1.25814 \\
\hline $3 d^{6}\left({ }^{5} z D\right) 4 p^{6} F_{7 / 2}$ & 2367.589 & 0.52374 & 0.28020 & 0.12867 & 1.28668 \\
\hline $3 d^{6}\left({ }^{5} z D\right) 4 p^{6} P_{7 / 2}$ & 2344.212 & 0.02559 & 0.04099 & 0.01882 & 1.88215 \\
\hline $3 \mathrm{~d}^{6}\left({ }^{5} \mathrm{zD}\right) 4 \mathrm{p}^{4} \mathrm{~F}_{9 / 2}$ & 2260.780 & 0.10173 & 3.43353 & 1.57668 & 1.57668 \\
\hline $3 d^{6}\left({ }^{5} z D\right) 4 p^{4} D_{7 / 2}$ & 2249.877 & 2.80641 & -2.23371 & -1.45873 & -1.45873 \\
\hline $3 d^{6}\left(a^{3} y F\right) 4 p^{4} F_{7 / 2}$ & 1611.200 & 0.39101 & -0.20865 & -0.09581 & -0.95813 \\
\hline $3 d^{5}\left({ }^{6} S\right) 4 s 4 p\left({ }^{3} y P\right){ }^{6} P_{7 / 2}$ & 1608.450 & 0.02487 & 0.01243 & -0.81818 & -2.18174 \\
\hline $3 d^{5}\left({ }^{6} S\right) 4 s 4 p\left({ }^{1} x P\right){ }^{6} P_{7 / 2}$ & 1260.533 & 2.04675 & 0.62672 & 0.52391 & 2.23911 \\
\hline $3 d^{5}\left({ }^{4} G\right) 4 s 4 p\left({ }^{3} y P\right){ }^{6} F_{11 / 2}$ & 1144.939 & 0.18342 & 0.19726 & 0.09058 & 2.05836 \\
\hline $3 d^{5}\left({ }^{4} G\right) 4 s 4 p\left({ }^{3} y P\right){ }^{6} F_{9 / 2}$ & 1143.224 & 0.20993 & 0.03515 & 0.01614 & 1.61395 \\
\hline $3 d^{5}\left({ }^{4} G\right) 4 s 4 p\left({ }^{3} y P\right){ }^{6} F_{7 / 2}$ & 1142.366 & 0.28012 & -0.25867 & -0.11878 & -1.18783 \\
\hline $3 d^{5}\left({ }^{4} \mathrm{P}\right) 4 s 4 p\left({ }^{3} \mathrm{P}\right){ }^{6} \mathrm{D}_{7 / 2}$ & 1260.533 & 0.22934 & 0.14743 & 0.06770 & 1.77017 \\
\hline $3 d^{6}\left({ }^{5} D\right) 5 p^{6} D_{9 / 2}$ & 1127.097 & 0.06211 & -0.00441 & -0.00203 & -1.02509 \\
\hline $3 d^{6}\left({ }^{5} D\right) 5 p^{6} D_{7 / 2}$ & 1125.446 & 0.05331 & 0.09425 & 0.04328 & 1.32792 \\
\hline $3 d^{5}\left({ }^{4} \mathrm{P}\right) 4 \mathrm{~s} 4 \mathrm{p}\left({ }^{3} \mathrm{P}\right){ }^{6} \mathrm{P}_{7 / 2}$ & 1121.975 & 0.24065 & -0.09503 & -0.04364 & -1.36368 \\
\hline $3 d^{6}\left({ }^{5} D\right) 5 p^{6} F_{11 / 2}$ & 1112.047 & 0.05395 & 1.10512 & 0.50747 & 1.07471 \\
\hline $3 d^{6}\left({ }^{5} D\right) 5 p^{4} F_{9 / 2}$ & 1106.362 & 2.24159 & -1.08932 & -0.50022 & -1.00217 \\
\hline $3 d^{6}\left({ }^{5} D\right) 5 p^{6} P_{7 / 2}$ & 1096.877 & 0.10028 & -0.17914 & -0.08226 & -1.22625 \\
\hline $3 d^{5}\left({ }^{4} \mathrm{D}\right) 4 \mathrm{~s} 4 \mathrm{p}\left({ }^{3} \mathrm{P}\right){ }^{6} \mathrm{~F}_{9 / 2}$ & 1083.419 & 0.24921 & -0.00481 & -0.00221 & -1.21026 \\
\hline $3 d^{5}\left({ }^{4} D\right) 4 s 4 p\left({ }^{3} P\right){ }^{6} F_{11 / 2}$ & 1081.874 & 0.25881 & 0.22132 & 0.10163 & 0.26558 \\
\hline $3 d^{5}\left({ }^{4} D\right) 4 s 4 p\left({ }^{3} P\right){ }^{6} D_{7 / 2}$ & 1063.971 & 0.16918 & -0.49919 & -0.22923 & -0.59903 \\
\hline $3 d^{5}\left({ }^{4} D\right) 4 s 4 p\left({ }^{3} P\right){ }^{6} D_{9 / 2}$ & 1063.176 & 0.82771 & -0.19849 & -0.09115 & -0.23819 \\
\hline $3 d^{5}\left({ }^{4} G\right) 4 s 4 p\left({ }^{3} w P\right){ }^{4} G_{9 / 2}$ & 1062.152 & 1.22393 & 0.72562 & 0.33320 & 0.87074 \\
\hline $3 d^{5}\left({ }^{4} \mathrm{D}\right) 4 \mathrm{~s} 4 \mathrm{p}\left({ }^{3} \mathrm{P}\right){ }^{6} \mathrm{P}_{7 / 2}$ & 1055.262 & 0.20848 & 1.03206 & 0.14320 & 0.85440 \\
\hline
\end{tabular}




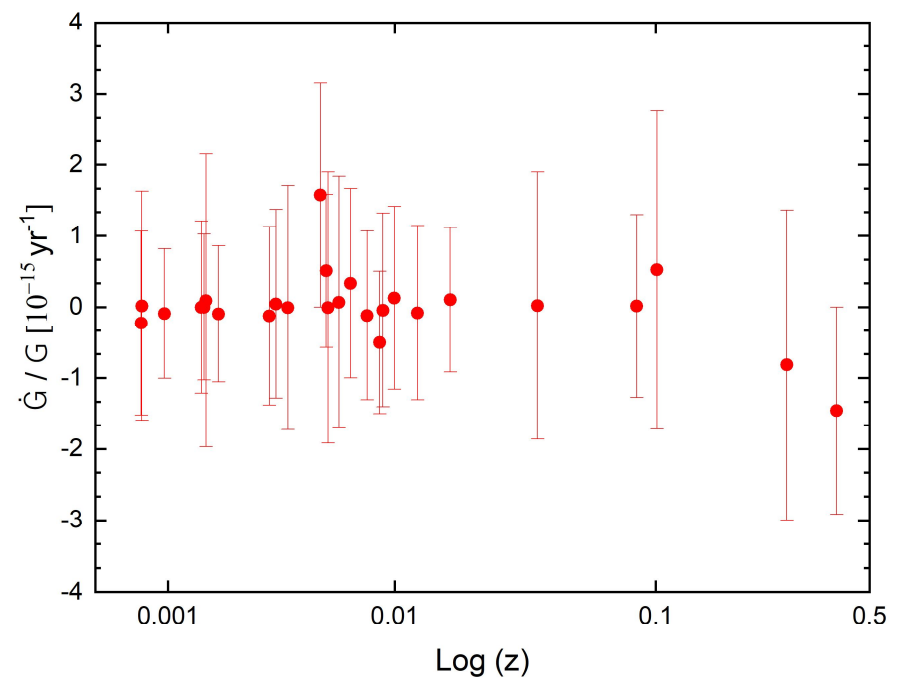

Figure 1. Illustration for the effect of time-variation of the gravitational constant, $\dot{G} / G$ with redshift.

Figure 1 represents the effect of time-variation of $G$ using 27 [Fe II] lines from observed quasar spectra combined with Ritz wavelengths. Each point of $\dot{G} / G$ is outlined by a point using minimal redshifts. The analysis procedure used the independent line ratios of $G$ with the shape lines of [Fe II], and has a small separation. As a result, we can estimate precisely the size of errors, including statistical and systematic errors, based on splitted-wavelengths for all line pairs of [Fe II] in an exact way. The presented analytical process was assigned $1 \sigma$ error, the same as in previous uses. Therefore, we can outline the best value of $\dot{G} / G$ with the used computation of the effect of time-variation on $\dot{G} / G$ versus $\chi^{2}$. In particular, the maximum change in $\dot{G} / G$ was determined by $\chi^{2}-\chi_{\min }^{2}=1$ with an error estimation as follows: $\Delta \chi^{2}=1$. Then, the minimal $\chi^{2}$ was used for each fit and applied for all fits with a standard deviation $\sigma_{t o t}^{2}=\sigma_{\dot{G} / G}^{2}+\sigma_{s y s}^{2}$ for the error estimation of the weighted mean.

\section{Discussion and Conclusion}

Astrophysical observations have provided a valuable tool for examining the variations of fundamental physical constants. Recently, studies focused on this topic have indicated that the effect of time-variation on the gravitational constant has different values at different timescales $[20,50]$. The study of pulsating white-dwarfs G117-B15A and R548 resulted in $\dot{G} / G \sim-1.8 \times$ $10^{-10} \mathrm{yr}^{-1}$ and $\dot{G} / G \sim-1.3 \times 10^{-10} \mathrm{yr}^{-1}$. The analysis used white dwarf asteroseismology, and found $\dot{G} / G=1.3 \times 10^{-10} \mathrm{yr}^{-1}$ [51-53]. This limit could be improved by applying the white cooling theory, such as $\dot{G} / G \leq 10^{-10}-10^{-11} \mathrm{yr}^{-1}$ [54], while other studies have reported $\dot{G} / G \sim-1.8 \times 10^{-12} \mathrm{yr}^{-1}$ by utilizing the galactic cluster NGC6791 [55]. Based on the pulsar binary of PSR1913+16 and Brans-Dicke theory, the different results gave $\dot{G} / G=(1.0 \pm 2.3) \times$ $10^{-11} \mathrm{yr}^{-1}$ and $\dot{G} / G=(1.0 \pm 2.3) \times 10^{-11} \mathrm{yr}^{-1}$. In particular, making use of a reexamination 
of this data and PSR B1855+09 data showed $\dot{G} / G=(4 \pm 5) \times 10^{-12} \mathrm{yr}^{-1}$ and $\dot{G} / G=(-9 \pm$ 18) $\times 10^{-12} \mathrm{yr}^{-1}$ [56-58]. Almost all updated results to present have only estimated this effect at a level of $10^{-12} \mathrm{yr}^{-1}$. There are $|\dot{G} / G|<1.6 \times 10^{-12} \mathrm{yr}^{-1}$ and $\dot{G} / G=(-0.6 \pm 4.2) \times$ $10^{-12} \mathrm{yr}^{-1}$ at $2 \sigma$ by applying data from six telescopes with p-mode spectra [59-63].

In this study, we provided a possible cosmological deviation of the gravitational constant over cosmic timescales. Our analysis yielded a critical result $\dot{G} / G=(0.918 \pm 2.830) \times 10^{-15} \mathrm{yr}^{-1}$ using the combined wavelengths of [Fe II] and Ritz wavelengths. The expected result is better than those achieved in previous studies applying Pulsar timing, Lunar Laser Ranging, Big Bang nucleosynthesis, and ages of globular clusters. Note that our study's obtained result from our study consisted of the most robust limit on the fine-structure constant and gravitational constant in various models, with extra dimensions as $\dot{G} / G \sim+10^{-15} \mathrm{yr}^{-1}$ [64]. Furthermore, this limit could be improved much more accuracy than current studies due to the best selection candidate, such as the $\mathrm{CH}_{3} \mathrm{OH}, \mathrm{OH}$, and $\mathrm{CH}$ molecules [65-70]. In general, the future analysis uses the quality of the data from high-resolution of astrophysical observations will provide the most robust estimate on upper bounds on a possible spatial and temporal variation of the gravitional constant.

Future laboratory tests and higher quality of the observational data will limit the spatial and temporal variations of the dimensionless physical constants. In any case, this study may be considered as a new tool of testing for parameters of unification scenarios [71].

\section{Declaration of Competing Interest}

The author declares no conflict of interest.

\section{Funding}

This research received no external funding.

\section{References}

[1] P. A. M. Dirac, Nature. 139, 323 (1937).

[2] J. Uzan, Rev. Mod. Phys.75, 403 (2003).

[3] J. Solà, Mod. Phys. Lett. A. 30, 22 (2015).

[4] Y.V. Stadnik, V.V. Flambaum, Phys. Rev. Lett. 115, 201301 (2015).

[5] H. Fritzsch, J. Solà, Mod. Phys. Lett. A. 30, 1540034 (2015)

[6] M. R. Wilczynska, et al,. Mon. Not. R. Astron. Soc. 454, 3082 (2015).

[7] T M. J. Duff, Contemp. Phys. 56, 35 (2015)

[8] A. Joyce et al., Phys. Rept. 568, 1 (2015).

[9] P. A. R. Ade et al., Astron. Astrophys. 580, A22 (2015).

[10] K. Masuda and Y. Suto, Publ. Astron. Soc. Japan. 68, L5 (2016).

[11] P. J. Mohr, D. B. Newell and B. N. Taylor, Rev. Mod. Phys. 88, 035009 (2016)

[12] R. I. Thompson, Mon. Not. R. Astron. Soc. 467, 4558 (2017).

[13] M. E. Mosquera and O. Civitarese, Phys. Rev. C. 96, 045802 (2017).

[14] V. A. Kostelecky and R. Lehnert, Phys. Rev. D. 63, 096002 (2001).

[15] M. C. D. Marsh, Phys. Rev. Lett. 118, 011302 (2017).

[16] H. Fritzsch, J. Sola and R. C. Nunes, Eur. Phys. J. C. 77, 193 (2017). 
[17] J. Solà, E. Karimkhani, A. Khodam-Mohammadi, Class. Quan. Grav. 34, 025006 (2017)

[18] J. K. Webb, et al., Phys. Rev. Lett. 107, 191101 (2011).

[19] T. D. Le, Astrophysics. 59, 285 (2016).

[20] C. J. A. P. Martins and A. M. M. Pinho, Phys. Rev. D. 95, 023008 (2017).

[21] C. J. A. P. Martins and A. M. M. Pinho, Phys. Rev. D. 95, 023008 (2017).

[22] L. Hart and J. Chluba, Mon. Not. Roy. Astron. Soc. 474, 1850 (2018).

[23] W. Zhao, B. S. Wright and B. Li, J. Cosm. Astropart. Phys. 052, 10 (2018).

[24] F. Hofmann, J. Mu"ller, and L. Biskupek, Astron. Astrophys. 522, L5 (2010).

[25] C. Yu et al., Ann. Phys. 531, 1800346 (2019).

[26] E. P. Bellinger and J. C. Dalsgaard, Astrophys. J. Lett. 887, L1 (2019).

[27] M. G. Kozlov and D. Budker, Ann. Phys. 10, 100254 (2018).

[28] E. Garcia-Berro, et al., Int. J. Mod. Phys. D. 15, 1163 (2006).

[29] O. G. Benvenuto, E. Garcia-Berro, and J. Isern, Phys. Rev. D. 69, 082002 (2004).

[30] J. Wu et al., Ann. Phys. 531, 1900013 (2019).

[31] W. W. Zhu et al., Mon. Not. Roy. Astron. Soc. 482, 3249 (2019).

[32] M. Jamil, E. N. Saridakis, and M. Setare, Phys. Lett. B. 679, 172 (2009).

[33] T. D. Le, Symmetry. 10, 722 (2018).

[34] T. D. Le, Results in Physics. 12, 1035 (2019).

[35] T. D. Le, Chinese Journal of Physics. 62, 252 (2019).

[36] L. G. Althaus, et al., Astron. Astrophys. 527, A72 (2011).

[37] C. J. A. P. Martins, Rep. Prog. Phys. 80, 126902 (2017).

[38] T. D. Le, Symmetry. 12, 344 (2020).

[39] A. Coc, et al., Phys. Rev. D. 76, 023511 (2007).

[40] T. D. Le, Gen. Relativ. Gravit. 53, 37 (2021).

[41] M. Aldenius, S. Johansson, M. T. Murphy, Mon. Not. Roy. Astron. Soc. 370, 444 (2006).

[42] G. Nave \& C. J. Sansonetti, Opt. Soc. Am. B. 28, 737 (2011).

[43] G. Nave, Mon. Not. Roy. Astron. Soc. 420, 1570 (2012).

[44] J. C. Pickering, et al., Mon. Not. Roy. Astron. Soc. 319, 163 (2000)

[45] J. C. Pickering, et al., Astron. Astrophys. 396, 715 (2002).

[46] X. Chen, S. P. Ellingsen \& Y. Mei, Res. Astron. Astrophys. 19, 018 (2019).

[47] S. A. Levshakov, et al., Mon. Not. Roy. Astron. Soc. 487, 5175 (2019).

[48] N. Prause and D. Reimers, Astron. Astrophys. 555, A88 (2013).

[49] A. Songaila and L. L. Cowie, Astrophys. J. 793, 103 (2014).

[50] J. B. Whitmore and M. T. Murphy, Mon. Not. R. Astron. Soc. 447, 446 (2015).

[51] C. M. Will, Living. Rev. Rel. 4, 4 (2001).

[52] E. García-Berro, et al., Int. J. Mod. Phys. D. 15, 1163 (2006).

[53] E. García-Berro, et al., Mem. S. A. It. 85, 118 (2014).

[54] E. García-Berro, et al., Mon. Not. Roy. Astron. Soc. 277, 801 (1995).

[55] E. García-Berro, et al., J. Cosm. Astropart. Phys. 5, 21 (2011).

[56] M. B. Bainbridge and J. K. Webb, Mon. Not. R. Astron. Soc. 468, 1639 (2017).

[57] M. Bainbridge and J. K. Webb, Universe. 3, 34 (2017)

[58] J. Ooba, K. Ichiki, T. Chiba, and N. Sugiyama, Prog. Theor. Exp. Phys. $043 E 03$ (2017).

[59] D. B. Guenther, L. M. Krauss \& P. Demarque, Astrophys. J. 498, 871 (1998).

[60] S. E. Thorsett, Phys. Rev. Lett. 77, 1432 (1996).

[61] J. D. Anderson, et al., Eur. Phys. Lett. 110, 10002 (2015). 
[62] E. P. Bellinger and J. Christensen-Dalsgaard, Astrophys. J. 887, L1 (2019).

[63] L. Jianbo, E. N. Saridakis, M. R. Setare, L. Xu, J. Cos. Astro. Phys. 31, 1003 (2020).

[64] P. Lorén-Aguilar, et al., Class. Quant. Grav. 20, 3885 (2003).

[65] A. Hees et al., Phys. Rev. Lett. 124, 081101 (2020).

[66] J. Alve, N. Sabti, M. Escudero and M. Fairbairn, Eur. Phys. J. C. 80, 148 (2020).

[67] S. Truppe, et al., Nature. Com. 4, 2600 (2013).

[68] T. L. Smith, D. Grin, D. Robinson and D. Qi, Phys. Rev. D. 99, 043531(2019).

[69] M. S. Safronova, Ann. Phys. 531, 1800364 (2019).

[70] M. R. Wilczynska, et al., Science Advances. 6, 9672 (2020).

[71] T. D. Le, Journal of High Energy Astrophysics. 29, 43 (2021). 
Figures

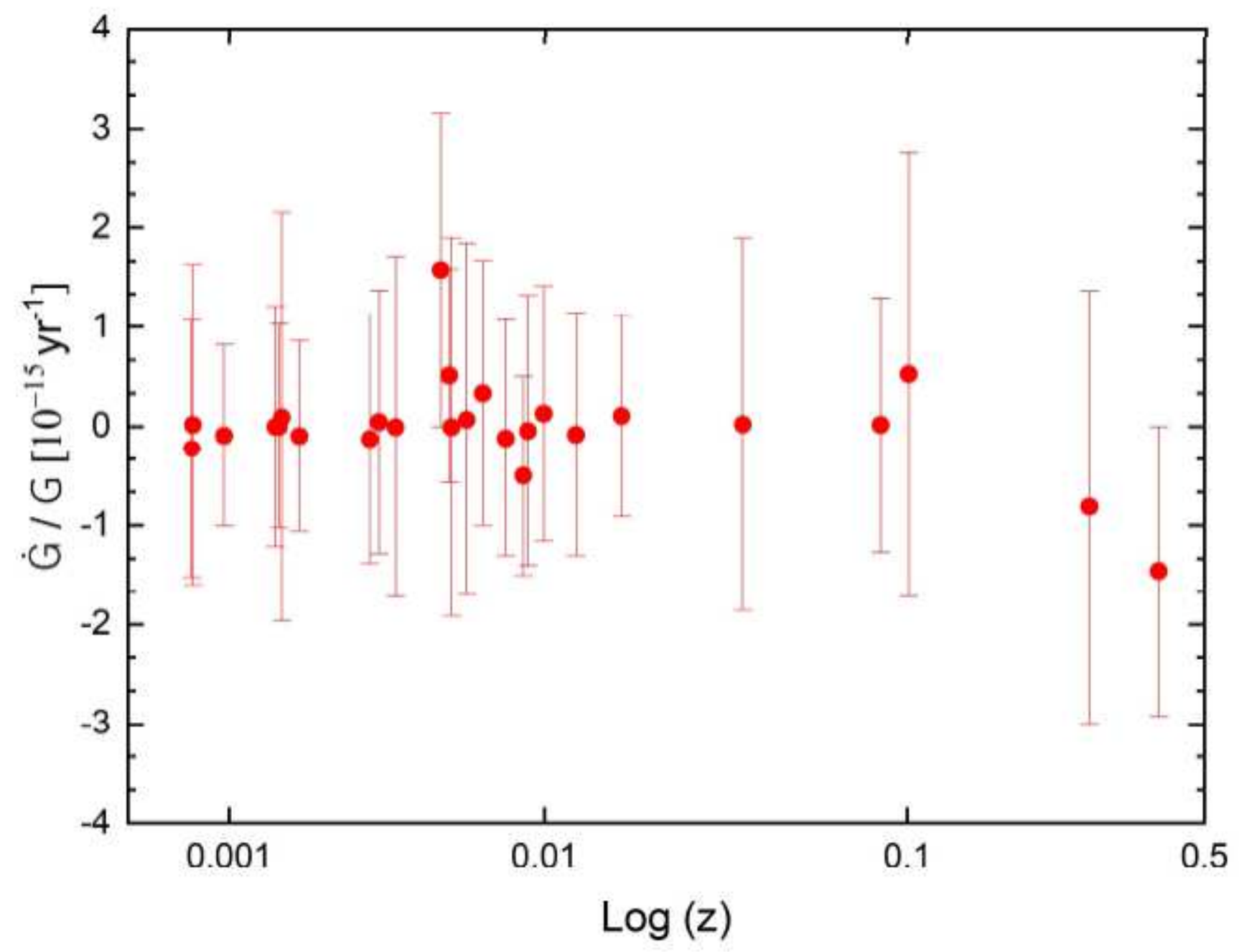

Figure 1

Illustration for the effect of time-variation of the gravitational constant, G/G with redshift. 